\title{
Request for Comments Summary
}

RFC Numbers 1000-1099

Status of This Memo

This memo is a slightly annotated list of the 100 RFCs from RFC 1000 through RFCs 1099. This is a status report on these RFCs. This memo provides information for the Internet community. It does not specify an Internet standard. Distribution of this memo is unlimited.

$\begin{array}{llll}\text { RFC } & \text { Author } & \text { Date } & \text { Title } \\ --- & ----- & --- & \\ & & & \\ 1099 & \text { Reynolds } & \text { Dec } 91 & \text { Requests For Comments Summary }\end{array}$

This memo.

1098 Case Apr 89 A Simple Network Management Protocol (SNMP)

This RFC is a re-release of RFC 1067, with a changed "Status of this Memo" section. This memo defines a simple protocol by which management information for a network element may be inspected or altered by logically remote users. In particular, together with its companion memos which describe the structure of management information along with the initial management information base, these documents provide a simple, workable architecture and system for managing TCP/IP-based internets and in particular the Internet.

1097 Miller Apr 89 Telnet Subliminal-Message Option

This RFC specifies a standard for the Internet community. Hosts on the Internet that display subliminal messages within the Telnet protocol are expected to adopt and implement this standard.

1096 Marcy Mar 89 Telnet X Display Location Option

This RFC specifies a standard for the Internet community. Hosts on the Internet that transmit the $\mathrm{X}$ display location within the Telnet protocol 
are expected to adopt and implement this standard.

1095 Warrier Apr 89 The Common Management Information Services and Protocol over TCP/IP (CMOT)

This memo defines a network management architecture that uses the International Organization for Standardization's (ISO) Common Management Information Services/Common Management Information Protocol (CMIS/CMIP) in a TCP/IP environment. This architecture provides a means by which control and monitoring information can be exchanged between a manager and a remote network element. In particular, this memo defines the means for implementing the Draft International Standard (DIS) version of CMIS/CMIP on top of Internet transport protocols for the purpose of carrying management information defined in the Internet-standard management information base. DIS CMIS/CMIP is suitable for deployment in TCP/IP networks while CMIS/CMIP moves toward becoming an International standard. Together with the relevant ISO standards and the companion RFCs that describe the initial structure of management information and management information base, these documents provide the basis for a comprehensive architecture and system for managing TCP/IPbased internets, and in particular the Internet.

1094 Sun Mar 89 NFS: Network File System Protocol Specification

This RFC describes a protocol that Sun Microsystems, Inc., and others are using. A new version of the protocol is under development, but others may benefit from the descriptions of the current protocol, and discussion of some of the design issues.

1093 Braun Feb 89 The NSFNET Routing Architecture

This document describes the routing architecture for the NSFNET centered around the new NSFNET Backbone, with specific emphasis on the interface between the backbone and its attached networks.

1092 Rekhter Feb 89 EGP and Policy Based Routing in the New NSFNET Backbone

This memo discusses implementation decisions for routing issues in the NSFNET, especially in the NSFNET Backbone. Of special concern is the restriction of routing information to advertize the best route as established by a policy decision. 
1091 VanBokkelen Feb 89 Telnet Terminal-Type Option

This RFC specifies a standard for the Internet community. Hosts on the Internet that exchange terminal type information within the Telnet protocol are expected to adopt and implement this standard.

This standard supersedes RFC 930. A change is made to permit cycling through a list of possible terminal types and selecting the most appropriate

1090 Ullmann Feb 89 SMTP on X.25

This memo proposes a standard for SMTP on the virtual circuit facility provided by the X.25 standard of the CCITT.

\section{Schoffstall Feb 89 SNMP over Ethernet}

This memo describes an experimental method by which the simple Network Management Protocol (SNMP) can be used over Ethernet MAC layer framing instead of the Internet UDP/IP protocol stack. This specification is useful for LAN based network elements that support no higher layer protocols beyond the MAC sub-layer.

1088 McLaughlin Feb $89 \quad \begin{aligned} & \text { A Standard for the Transmission of IP } \\ & \text { Datagrams over NetBIOS Networks }\end{aligned}$

This document specifies a standard method of encapsulating the Internet Protocol (IP) datagrams on NetBIOS networks.

1087 IAB Jan 89 Ethics and the Internet

This memo is a statement of policy by the Internet Activities Board (IAB) concerning the proper use of the resources of the Internet.

1086 Onions Dec 88 ISO-TPO bridge between TCP and X.25

This memo proposes a standard for the Internet community. Hosts on the Internet that choose to implement ISO TPO transport connectivity between TCP and X.25 based hosts are expected to experiment with this proposal. TCP port 146 is reserved for this proposal. 
1085 Rose Dec 88 ISO Presentation Services on top of TCP/IP-based internets

RFC 1006 describes a mechanism for providing the ISO transport service on top of TCP/IP. Once this method is applied, one may implement "real" ISO applications on top of TCP/IP-based internets, by simply implementing OSI session, presentation, and application services on top of the transport service access point which is provided on top of the TCP. Although straight-forward, there are some environments in which the richness provided by the OSI application layer is desired, but it is nonetheless impractical to implement the underlying osI infrastructure (i.e., the presentation, session, and transport services on top of the $\mathrm{TCP})$.

This memo describes an approach for providing "stream-lined" support of OSI application services on top of TCP/IP-based internets for such constrained environments. This memo proposes a standard for the Internet community.

1084 Reynolds Dec 88 BOOTP Vendor Information Extensions

This RFC is a slight revision and extension of RFC-1048 by Philip Prindeville. This memo will be updated as additional tags are are defined. This edition introduces Tag 13 for Boot File Size. Comments and suggestions for improvements are sought.

1083 IAB Dec 88 IAB Official Protocol Standards

This memo describes the state of standardization of protocols used in the Internet as determined by the Internet Activities Board (IAB). An overview of the standards procedures is presented first, followed by discussions of the standardization process and the RFC document series, then the explanation of the terms is presented, the lists of protocols in each stage of standardization follows, and finally pointers to references and contacts for further information.

This memo is issued quarterly, please be sure the copy you are reading is dated within the last three months.

1082 Rose Nov 88 Post Office Protocol - Version 3 Extended Service Offerings

This memo suggests a simple method for workstations to dynamically access mail from a discussion group server, as an extension to an earlier memo which dealt with dynamically accessing mail from a mailbox 
server using the Post Office Protocol - Version 3 (POP3). This RFC specifies a proposed protocol for the Internet community, and requests discussion and suggestions for improvements. All of the extensions described in this memo to the POP3 are OPTIONAL.

1081 Rose Nov 88 Post Office Protocol - Version 3

This memo suggests a simple method for workstations to dynamically access mail from a mailbox server. This RFC specifies a proposed protocol for the Internet community, and requests discussion and suggestions for improvements.

1080 Hedrick Dec 88 Telnet Remote Flow Control Option

This RFC specifies a standard for the Internet community. Hosts on the Internet that do remote flow control within the Telnet protocol are expected to adopt and implement this standard.

1079 Hedrick Dec 88 Telnet Terminal Speed Option

This RFC specifies a standard for the Internet community. Hosts on the Internet that exchange terminal speed information within the Telnet protocol are expected to adopt and implement this standard.

1078 Lottor Nov 88 TCP Port Service Multiplexer (TCPMUX)

This RFC proposes an Internet standard which can be used by future TCP services instead of using 'well-known ports'.

1077 Leiner Nov 88 Critical Issues in High Bandwidth Networking

This memo presents the results of a working group on High Bandwidth Networking. This RFC is for your information and you are encouraged to comment on the issues presented.

1076 Trewitt Nov 88 HEMS Monitoring and Control Language

This RFC specifies a query language for monitoring and control of network entities. This RFC supercedes RFC 1023, extending the query language and providing more discussion of the underlying issues. 
This language is a component of the High-Level Entity Monitoring System (HEMS) described in RFC 1021 and RFC 1022. Readers may wish to consult these RFCs when reading this memo. RFC 1024 contains detailed assignments of numbers and structures used in this system. Portions of RFC 1024 that define query language structures are superceded by definitions in this memo. This memo assumes a knowledge of the Iso data encoding standard, ASN.1.

1075 Waitzman Nov 88 Distance Vector Multicast Routing Protocol

This RFC describes a distance-vector-style routing protocol for routing multicast datagrams through an internet. It is derived from the Routing Information Protocol (RIP), and implements multicasting as described in RFC-1054. This is an experimental protocol, and its implementation is not recommended at this time.

1074 Rekhter Oct 88

The NSFNET Backbone SPF based Interior Gateway Protocol

This RFC is an implementation description of the standard ANSI IS-IS and ISO ES-IS routing protocols within the NSFNET backbone network.

1073 Waitzman Oct 88 Telnet Window Size Option

This RFC describes a proposed Telnet option to allow a client to convey window size to a Telnet server.

1072 Jacobson Oct 88 TCP Extensions for Long-Delay Paths

This RFC proposes a set of extensions to the TCP protocol to provide efficient operation over a path with a high bandwidth*delay product. These extensions are not proposed as an Internet standard at this time. Instead, they are intended as a basis for further experimentation and research on transport protocol performance.

1071 Braden Aug 88 Computing the Internet Checksum

This RFC summarizes techniques and algorithms for efficiently computing the Internet checksum. It is not a standard, but a set of useful implementation techniques. 
1070 Hagens Feb 8

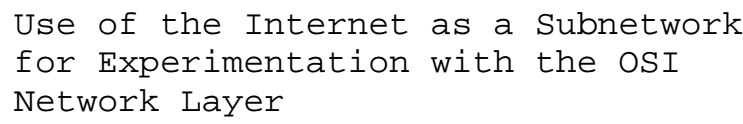

This RFC proposes a scenario for experimentation with the International Organization for Standardization (ISO) Open systems Interconnection (OSI) network layer protocols over the Internet and requests discussion and suggestions for improvements to this scenario. This RFC also proposes the creation of an experimental OSI internet. To participate in the experimental OSI internet, a system must abide by the agreements set forth in this RFC.

1069 Callon Feb 89

Guidelines for the use of Internet-IP addresses in the ISO ConnectionlessMode Network Protocol

This RFC suggests an addressing scheme for use with the ISO Connectionless Network Protocol (CLNP) in the Internet. This is a solution to one of the problems inherent in the use of "ISO-grams" in the Internet. This memo is a revision of RFC 986. This RFC suggests a proposed protocol for the Internet community, and requests discussion and suggestions for improvements.

1068 DeSchon Aug 8 Background File Transfer Program (BFTP)

This RFC describes an Internet background file transfer service that is built upon the third-party transfer model of FTP. No new protocols are involved. The purpose of this memo is to stimulate discussions on new Internet service modes.

1067 Case Aug 88 A Simple Network Management Protocol

This RFC defines a simple protocol by which management information for a network element may be inspected or altered by logically remote users. In particular, together with its companion memos which describe the structure of management information along with the initial management information base, these documents provide a simple, workable architecture and system for managing TCP/IP-based internets and in particular, the Internet.

This memo specifies a draft standard for the Internet community. TCP/IP implementations in the Internet which are network manageable are expected to adopt and implement this specification. 
This RFC provides the initial version of the Management Information Base (MIB) for use with network management protocols in TCP/IP-based internets in the short-term. In particular, together with its companion memos which describe the structure of management information along with the initial network management protocol, these documents provide a simple, workable architecture and system for managing TCP/IP-based internets, and in particular, the Internet.

This memo specifies a draft standard for the Internet community. TCP/IP implementations in the Internet which are network manageable are expected to adopt and implement this specification.

1065 Rose Aug 88

Structure and Identification of Management Information for TCP/IP-based internets

This RFC provides the common definitions for the structure and identification of management information for TCP/IP-based internets. In particular, together with its companion memos, which describe the initial management information base along with the initial network management protocol, these documents provide a simple, working architecture and system for managing TCP/IP-based internets and in particular, the Internet.

This memo specifies a draft standard for the Internet community. TCP/IP implementation in the Internet which are network manageable are expected to adopt and implement this specification.

1064 Crispin Jul 88 Interactive Mail Access Protocol Version 2

This memo suggests a method for workstations to dynamically access mail from a mailbox server ("respository"). This RFC specifies a standard for the SUMEX-AIM community and a proposed experimental protocol for the Internet community. Discussion and suggestions for improvement are requested.

1063 Mogul Jul 88 IP MTU Discovery Options

A pair of IP options that can be used to learn the minimum MTU of a path through an internet is described, along with its possible uses. This is a proposal for an Experimental protocol. 
$1062 \quad$ Romano Aug 88 Internet Numbers

This memo is an official status report on the network numbers and gateway autonomous system numbers used in the Internet community.

1061 Never issued.

1060 Reynolds Mar $90 \quad$ Assigned Numbers

This memo is a status report on the parameters (i.e., numbers and keywords) used in protocols in the Internet community. Distribution of this memo is unlimited.

1059 Mils Jul 88 Network Time Protocol (Version 1) Specification and Implementation

This memo describes the Network Time Protocol (NTP), specifies its formal structure and summarizes information useful for its implementation. NTP provides the mechanisms to synchronize time and coordinate time distribution in a large, diverse internet operating at rates from mundane to lightwave. It uses a returnable-time design in which a distributed subnet of time servers operating in a selforganizing, hierarchical master-slave configuration synchronizes logical clocks within the subnet and to national time standards via wire or radio. The servers can also redistribute reference time via local routing algorithms and time daemons.

The NTP architectures, algorithms and protocols which have evolved over several years of implementation and refinement are described in this document. The prototype system, which has been in regular operation in the Internet for the last two years, is described in an Appendix along with performance data which shows that timekeeping accuracy throughout most portions of the Internet can be ordinarily maintained to within a few tens of milliseconds, even the cases of failure or disruption of clocks, time servers or nets. This is a Draft standard for an Elective protocol.

1058 Hedrick Jun 88 Routing Information Protocol

This RFC describes an existing protocol for exchanging routing information among gateways and other hosts. It is intended to be used as a basis for developing gateway software for use in the Internet community. 
1057 Sun Jun 88

RPC: Remote Procedure Call Protocol Specification Version 2

This RFC describes a standard that Sun Microsystems and others are using, and is one we wish to propose for the Internet's consideration. This memo is not an Internet standard at this time.

1056 Lambert Jun 88 PCMAIL: A Distributed Mail System for Personal Computers

This memo is a discussion of the Pcmail workstation based distributed mail system. It is identical to the discussion in RFC-993, save that a new, much simpler mail transport protocol is described. The new transport protocol is the result of continued research into ease of protocol implementation and use issues.

1055 Romkey Jun 88 A Nonstandard for Transmission of IP Datagrams over Serial Lines: SLIP

The TCP/IP protocol family runs over a variety of network media: IEEE 802.3 (ethernet) and 802.5 (token ring) LAN's, X.25 lines, satellite links, and serial lines. There are standard encapsulations for IP packets defined for many of these networks, but there is no standard for serial lines. SLIP, Serial Line IP, is a currently a de facto standard, commonly used for point-to-point serial connections running TCP/IP. It is not an Internet standard.

1054 Deering May 88 Host Extensions for IP Multicasting

This memo specifies the extensions required of a host implementation of the Internet Protocol (IP) to support multicasting. IP multicasting is the transmission of an IP datagram to a "host group", a set hosts identified by a single IP destination address. A multicast datagram is delivered to all members of its destination host group with the same "best-efforts" reliability as regular unicast IP datagrams. It is proposed as a standard for IP multicasting in the Internet. This specification is a major revision of $\mathrm{RFC}-988$.

1053 Levy Apr 88 Telnet X.3 PAD Option

This RFC proposes a new option to Telnet for the Internet community, and requests discussion and suggestions for improvements. 
IAB Recommendations for the Development of Internet Network Management Standards

This RFC is intended to convey to the Internet community and other interested parties the recommendations of the Internet Activities Board (IAB) for the development of network management protocols for use in the TCP/IP environment. This memo does NOT, in and of itself, define or propose an Official Internet Protocol. It does reflect, however, the policy of the IAB with respect to further network management development in the short and long term.

$1051 \quad$ Prindevile Mar 88

A Standard for the Transmission of IP Datagrams and ARP Packets over ARCNET Networks

This memo specifies a standard method of encapsulating Internet Protocol (IP) and Address Resolution Protocol (ARP) datagrams on an ARCNET. This RFC is a standard protocol for the Internet community.

$\begin{aligned} 1050 \text { Sun Apr 88 } & \text { RPC: Remote Procedure Call } \\ & \text { Protocol Specification }\end{aligned}$

This memo specifies a message protocol used in implementing Sun's Remote Procedure Call (RPC) package. This RFC describes a standard that sun Microsystems and others are using and is one they wish to propose for the Internet's consideration. It is not an Internet standard at this time.

1049 Sirbu Mar 88 A Content-Type Header Field for Internet Messages

This memo suggests proposed additions to the Internet Mail Protocol, RFC-822, for the Internet community, and requests discussion and suggestions for improvements.

1048 Prindevile Feb 88 BOOTP Vendor Information Extensions

This memo proposes an addition to the Bootstrap Protocol (BOOTP). Comments and suggestions for improvements are sought. 
1047 Partridge Feb $88 \quad$ Duplicate Messages and SMTP

An examination of a synchronization problem in the Simple Mail Transfer Protocol (SMTP) is presented. This synchronization problem can cause a message to be delivered multiple times. A method for avoiding this problem is suggested. Nodding familiarity with the SMTP specification, $\mathrm{RFC}-821$, is required.

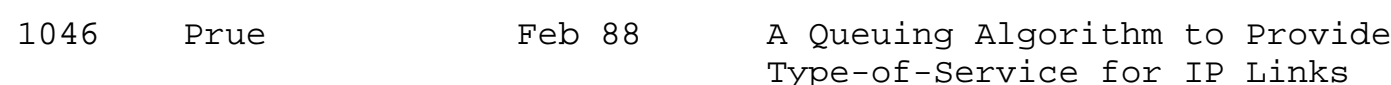

This memo is intended to explore how Type-of-Service might be implemented in the Internet. The proposal describes a method of queuing which can provide the different classes of service. The technique also prohibits one class of service from consuming excessive resources or excluding other classes of service. This is an "idea paper" and discussion is strongly encouraged.

1045 Cheriton Feb 88 VMTP: Versatile Message Transaction Protocol Protocol Specification

This memo specifies the Versatile Message Transaction Protocol (VMTP) [Version 0.7 of 19-Feb-88], a transport protocol specifically designed to support the transaction model of communication, as exemplified by remote procedure call (RPC). The full function of VMTP, including support for security, real-time, asynchronous message exchanges, streaming, multicast and idempotency, provides a rich selection to the VMTP user level. Subsettability allows the VMTP module for particular clients and servers to be specialized and simplified to the services actually required. Examples of such simple clients and servers include PROM network bootload programs, network boot servers, data sensors and simple controllers, to mention but a few examples. This RFC describes a protocol proposed as a standard for the Internet community.

1044 Hardwick Feb 88

Internet Protocol on Network Systems HYPERchannel Protocol Specification

This memo intends to provide a complete discussion of the protocols and techniques used to embed DoD standard Internet Protocol datagrams (and its associated higher level protocols) on Network systems Corporation's HYPERchannel equipment. This document is directed toward network planners and implementors who are already familiar with the TCP/IP protocol suite and the techniques used to carry TCP/IP traffic on common networks such as the DDN or the Ethernet. No great familiarity with NSC products is assumed; an appendix is devoted to a review of NSC 
technologies and protocols.

1043 Yasuda Feb 88 TELNET Data Entry Terminal Option

DODIIS Implementation

This RFC suggests a proposed protocol on the TELNET Data Entry Terminal (DET) Option - DODIIS Implementation for the Internet community. It is intended that this specification be capatible with the specification of DET Option in RFC-732. Discussion and suggests for improvements are encouraged.

1042 Postel Feb 88 A Standard for the Transmission of IP Datagrams over IEEE 802 Networks

This RFC specifies a standard method of encapsulating the Internet Protocol (IP) datagrams and Address Resolution Protocol (ARP) requests and replies on IEEE 802 Networks to allow compatible and interoperable implementations. This RFC specifies a protocol standard for the Internet community.

1041 Rekhter Jan 88 Telnet 3270 Regime Option

This RFC specifies a proposed standard for the Internet community. Hosts on the Internet that want to support 3270 data stream within the Telnet protocol, are expected to adopt and implement this standard.

1040 Linn Jan 88 Privacy Enhancement for Internet Electronic Mail: Part I Message Encipherment and Authentication Procedures

This RFC is the Outgrowth of a series of IAB Privacy Task Force meetings and of internal working papers distributed for those meetings. This memo defines message encipherment and authentication procedures, as the initial phase of an effort to provide privacy enhancement services for electronic mail transfer in the Internet. Detailed key management mechanisms to support these procedures will be defined in a subsequent RFC. As a goal of this initial phase, it is intended that the procedures defined here be compatible with a wide range of key management approaches, including both conventional (symmetric) and public-key (asymmetric) approaches for encryption of data encrypting keys. Use of conventional cryptography for message text encryption and/or integrity check computation is anticipated. 
1039 Latham Jan 88

A DoD Statement on Open Systems Interconnection Protocols

This RFC reproduces a memorandum issued on 2-JUL-87 from the Assistant Secretary of Defense for Command, Control, Communications, and Intelligence (ASDC31) to the Director of the Defense Communications Agency (DCA). This memo is distributed for information only.

1038 St. Johns Jan 88 Draft Revised IP Security Option

This memo is a pre-publication draft of the revised Internet Protocol Security option. This RFC reflects the version as approved by the Protocol standards steering group, and is provided for informational purposes only. The final version of this document will be available from Navy publications and should not differ from this document in any major fashion. This document will be published as a change to the MILSTD 1777, "Internet Protocol".

1037 Greenberg Dec 87 NFILE - A File Access Protocol

This document includes a specification of the NFILE file access protocol and its underlying levels of protocol, the Token List Transport Layer and Byte Stream with Mark. The goal of this specification is to promote discussion of the ideas described here, and to encourage designers of future file protocols to take advantage of these ideas. A secondary goal is to make the specification available to sites that might benefit from implementing NFILE.

1036 Horton Dec 87 Standard for Interchange of USENET Messages

This RFC defines the standard format for the interchange of network News messages among USENET hosts. It updates and replaces RFC-850, reflecting version B2.11 of the News program. This memo is distributed as an RFC to make this information easily accessible to the Internet community. It does not specify an Internet standard.

1035 Mockapetris Nov 87 Domain Names - Implementation Specification

This RFC is the revised specification of the protocol and format used in the implementation of the Domain Name system. It obsoletes RFC-883. This memo documents the details of the domain name client - server communication. 
Domain Names - Concepts and Facilities

This RFC is the revised basic definition of The Domain Name system. It obsoletes RFC-882. This memo describes the domain style names and their used for host address look up and electronic mail forwarding. It discusses the clients and servers in the domain name system and the protocol used between them.

1033 Lottor Nov 87 Domain Administrators Operations Guide

This RFC provides guidelines for domain administrators in operating a domain server and maintaining their portion of the hierarchical database. Familiarity with the domain system is assumed (see RFCs 1031, 1032 , 1034, and 1035).

1032 Stahl Nov 87 Domain Administrators Guide

Domains are administrative entities that provide decentralized management of host naming and addressing. The domain-naming system is distributed and hierarchical.

This memo describes procedures for registering a domain with the Network Information Center (NIC) of Defense Data Network (DDN), and offers guidelines on the establishment and administration of a domain in accordance with the requirements specified in RFC-920. It is recommended that the guidelines described in this document be used by domain administrators in the establishment and control of second-level domains. The role of the domain administrator (DA) is that of coordinator, manager, and technician. If his domain is established at the second level or lower in the tree, the domain administrator must register by interacting with the management of the domain directly above this.

1031 Lazear Nov 87 Milnet Name Domain Transition

This RFC consolidates information necessary for the implementation of domain style names throughout the DDN/MILNET Internet community. The introduction of domain style names will impact all hosts in the DDN/MILNET Internet. This RFC is designed as an aid to implementors and administrators by providing: 1) an overview of the transition process from host tables to domains, 2) a timetable for the transition, and 3) references to documentation and software relating to the domain system. 
This memo describes the results gathered from testing NETBLT over three networks of different bandwidths and round-trip delays. The results are not complete, but the information gathered so far has not been promising. The NETBLT protocol is specified in RFC-998; this document assumes an understanding of the specification as described in RFC-998.

1029 Parr

May 88

\author{
A More Tolerant Approach to Address \\ Resolution for Multi-Lan system of \\ Ethernets
}

This memo discusses an extension to a Bridge Protocol to detect and disclose changes in heighbouring host address parameters in a Multi-Lan system of Ethernets. The problem is one which is appearing more and more regularly as the interconnected systems grow larger on Campuses and in Commercial Institutions. This RFC suggests a protocol enhancement for the Internet community, and requests discussion and suggestions for improvements.
1028
Davin
Nov 87
A Simple Gateway Monitoring Protocol

This memo defines a simple application-layer protocol by which management information for a gateway may be inspected or altered by remote users. This proposal is intended only as an interim response to immediate gateway monitoring needs.

1027 Carl-Mitchell Oct 87 Using ARP to Implement Transparent Subnet Gateways

This RFC describes the use of the Address Resolution Protocol (ARP) by subnet gateways to permit hosts on the connected subnets to communicate without being aware of the existence of subnets, using the technique of "Proxy ARP".

1026 Kille Sep 87 Addendum to RFC-987 (Mapping between $\mathrm{X} .400$ and $\mathrm{RFC}-882$ )

This memo suggest a proposed protocol for the Internet community, and request discussion and suggestions for improvements. 
1025 Postel Sep $87 \quad$ TCP and IP Bake off

This memo describes some of the procedures, scoring and tests used in the TCP and IP bake offs held in the early development of these protocols. These procedures and tests may still be of use in testing newly implemented TCP and IP modules.

$1024 \quad$ Partridge Oct $87 \quad$ HEMS Variable Definitions

This memo assigns instruction codes, defines object formats and object semantics for use with the High-Level Monitoring and Control Language, defined in RFC-1023. A general system has been described in previous memos (RFC-1021, RFC-1022). This system is called the High-Level Entity Management system (HEMS). This memo is provisional and the definitions are subject to change. Readers should confirm with the authors that they have the most recent version. This RFC assumes a working knowledge of the ISO data encoding standard, ASN.1, and a general understanding of the IP protocol suite.

1023 Partridge Oct 87 HEMS Monitoring and Control Language

This RFC specifies the High-Level Entity Management System (HEMS)

Monitoring and Control Language. This language defines the requests and replies used in HEMS. This memo assumes knowledge of the HEMS system described in RFC-1021, and of the ISO data encoding standard, ASN.1.

1022 Partridge Oct 87 The High-Level Entity Management Protocol (HEMP)

This memo presents an application protocol for managing network entities such as hosts, gateways, and front end machines. This protocol is a component of the High-level Entity Management System HEMS), described is RFC-1021. This memo also assumes a knowledge of the ISO data encoding standard, ASN.1.

1021 Partridge Oct 87 The High-Level Entity Management System (HEMS)

This memo provides a general overview of the High-level Entity management system (HEMS). This system is experimental, and is currently being tested in portions of the Internet. 
1020 Romano Nov $87 \quad$ Internet Numbers

This RFC is a list of the Assigned IP Network Numbers and EGP Autonomous System Numbers. This RFC obsoletes RFC-997.

1019 Arnon Sep 87 Report of the Workshop on

Environments for Computational

Mathematics

This memo is a report on the discussion of the representation of equations in a workshop at the ACM SIGGRAPH Conference held in Anaheim, California on 30 July 1987.

$1018 \quad$ McKenzie Aug 87 Some Comments on SQuID

This memo is a discussion of some of the ideas expressed in RFC-1016 on Source Quench. This memo introduces the distinction of the cause of congestion in a gateway between the effects of "Funneling" and Mismatch". It is offered in the same spirit as RFC-1016; to stimulate discussion. The opinions offered are personal, not corporate, opinions.

1017 Leiner Aug 87 Network Requirements for Scientific Research

This RFC identifies the requirements on communication networks for supporting scientific research. It proposes some specific areas for near term work, as well as some long term goals. This is an "idea" paper and discussion is strongly encouraged.

1016 Prue July 87 Something a Host Could Do with Source Quench: The Source Quench Introduced Delay (SQuID)

The memo is intended to explore the issue of what a host could do with a source quench. The proposal is for each source host IP module to introduce some delay between datagrams sent to the same destination host. This is a "crazy idea paper" and discussion is essential.

1015 Leiner July 87 Implementation Plan for Interagency Research Internet

This RFC proposes an Interagency Research Internet as the natural outgrowth of the current Internet. This is an "idea paper" and 
discussion is strongly encouraged.

1014 Sun Jun 87 XDR: External Data Representation Standard

XDR is a standard for the description and encoding of data. It is useful for transferring data between different computer architectures. XDR fits into ISO presentation layer, and is roughly analogous in purpose to X.409, ISO Abstract Syntax Notation. The major difference between these two is that XDR uses implicit typing, while X.409 uses explicit typing. This RFC is distributed for information only, it does not establish a Internet standard.

1013 Scheifler Jun 87 X Window System Protocol, Version 11-Beta

This RFC is distributed to the Internet community for information only. It does not establish an Internet standard. The $\mathrm{X}$ window system has been widely reviewed and tested. The Internet community is encouraged to experiment with it.

1012 Reynolds Jun $87 \quad$ Bibliography of Requests for Comments 1-999

This RFC is a reference guide for the Internet community which provides a bibliographic summary of the Request for Comments numbers 1 through 999 issued between the years 1969-1987.

1011 Reynolds May 87 Official Internet Protocols

This memo is an official status report on the protocols used in the Internet community. It identifies the documents specifying the official protocols used in the Internet. Comments indicate any revisions or changes planned.

$1010 \quad$ Reynolds May $87 \quad$ Assigned Numbers

This memo is an official status report on the numbers used in protocols in the Internet community. It documents the currently assigned values from several series of numbers including link, socket, port, and protocol, used in network protocol implementations. 
1009 Braden Jun 87 Requirements for Internet Gateways

This RFC summarizes the requirements for gateways to be used between networks supporting the Internet protocols. This document is a formal statement of the requirements to be met by gateways used in the Internet system. As such, it is an official specification for the Internet community.

1008

$\mathrm{McCOY}$

Jun 87

Implementation Guide for the ISO Transport Protocol

This RFC is being distributed to members of the Internet community in order to solicit comments on the Implementors Guide. While this document may not be directly relevant to the research problems of the Internet, it may be of some interest to a number of researchers and implementors.

1007 McCoy Jun 87 Military Supplement to the ISO Transport Protocol

This document supplements the Transport Service and Protocol of the International Standards Organization (ISO), IS 8072 and IS 8073, respectively, and their formal descriptions by providing conventions, option selections and parameter values. This RFC is being distributed to members of the Internet community in order to solicit comments on the Draft Military Supplement. While this document may not be directly relevant to the research problems of the Internet, it may be of some interest to a number of researchers and implementors.

1006 Rose May 87 ISO Transport Services on top of the TCP Version: 3

This memo specifies a standard for the Internet community. Hosts on the Internet that choose to implement ISO transport services on top of the TCP are expected to adopt and implement this standard. TCP port 102 is reserved for hosts which implement this standard. This memo specifies version 3 of the protocol and supersedes RFC-983. Changes between the protocol is described in $\mathrm{RFC}-983$ and this memo are minor, but unfortunately incompatible.

1005 Khanna May 87 The ARPANET AHIP-E Host Access Protocol (Enhanced AHIP)

This RFC is a proposed specification for the encoding of Class A IP 
addresses for use on ARPANET-style networks such as the Milnet and Arpanet, and for enhancements to the ARPANET AHIP Host Access Protocol (AHIP; formerly known as 1822). These enhancements increase the size of the PSN field, allow ARPANET hosts to use logical names to address each other, allow for the communication of type-of-service information from the host to the PSN and enable the PSN to provide congestion feedback to the host on a connection basis.

$1004 \quad$ Mils Apr $87 \quad \begin{aligned} & \text { A Distributed-Protocol } \\ & \text { Authentication Scheme }\end{aligned}$

The purpose of this RFC is to focus discussion on authentication problems in the Internet and possible methods of solution. The proposed solutions this document are not intended as standards for the Internet at this time. Rather, it is hoped that a general consensus will emerge as to the appropriate solution to authentication problems, leading eventually to the adoption of standards. This document suggests mediated access-control and authentication procedures suitable for those cases when an association is to be set up between users belonging to different trust environments.

$1003 \quad$ Katz Mar 87 Issues in Defining an Equations Representation Standard

This memo is intended to identify and explore issues in defining a standard for the exchange of mathematical equations. No attempt is made at a complete definition and more questions are asked than are answered. Questions about the user interface are only addressed to the extent that they affect interchange issues.

1002 NETBIOS Mar 87 Protocol standard for A NetBIOS Service on a TCP/UDP Transport: Detailed Specifications

This RFC defines a proposed standard protocol to support NetBIOS services in a TCP/IP environment. Both local network and internet operation are supported. Various node types are defined to accommodate local and internet topologies and to allow operation with or without the use of IP broadcast. This RFC gives the detailed specifications of the netBIOS-over-TCP packets, protocols, and defined constants and variables. A more general overview is found in a companion RFC, "Protocol Standard For NetBIOS Service on TCP/UDP Transport: Concepts and Methods". 
Protocol Standard for A NetBIos Service on a TCP/UDP Transport: Concepts and Methods

This RFC defines a proposed standard protocol to support NetBIOS services in a TCP/IP environment. Both local network and internet operation are supported. Various node types are defined to accommodate local and internet topologies and to allow operation with or without the use of IP broadcast. This RFC describes the NetBIOS-over-TCP protocols in a general manner, emphasizing the underlying ideas and techniques. Detailed specifications are found in a companion RFC, "Protocol Standard For a NetBIOS Service on a TCP/UDP Transport: Detailed Specifications".

$1000 \quad$ Reynolds Aug $87 \quad \begin{aligned} & \text { The Request for Comments Reference } \\ & \text { Guide }\end{aligned}$

This RFC Reference Guide is intended to provide a historical account by categorizing and summarizing of the Request for Comments numbers 1 through 999 issued between the years 1969-1987. These documents have been crossed referenced to indicate which RFCs are current, obsolete, or revised.

Security Considerations

Security issues are not discussed in this memo.

Author's Address

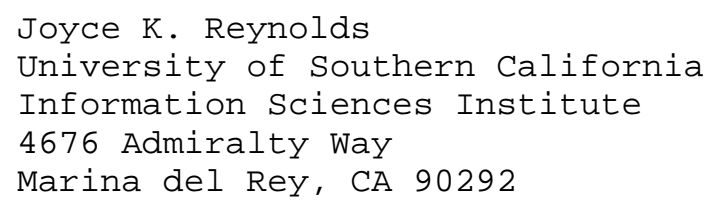

EMail: JKREYCISI.EDU 